International conference on diagnostic pathology

of breast and female genital tract. Sri Lanka 2016

\title{
Expression of epithelial and mesenchymal markers in invasive (ductal) breast carcinoma and nodal metastasis
}

\author{
L.Y. V. Pathirana' ${ }^{1}$ E. H. Siriweera ${ }^{2}$, H. A. Amaratunga ${ }^{1}$, S. B. Adikari ${ }^{1}$ and N. P. A. D. \\ Gunasinghe ${ }^{1}$
}

Department of Anatomy, Faculty of Medicine, University of Peradeniya, Sri Lanka ${ }^{1}$

Department of Pathology, Faculty of Medicine, University of Peradeniya, Sri Lanka ${ }^{2}$

DOI: http://doi.org/10.4038/jdp.v11i2.7710

\section{Introduction}

The phenomenon, Epithelial-mesenchymal transition (EMT) has been shown to enable epithelial cells to acquire a mesenchymal phenotype facilitating cancer cell mobilization. Evidence in favour has been derived predominantly from cancer cell lines and/or animal model studies. The objectives of this study were to observe E-cadherin and vimentin expression patterns in invasive (ductal) breast carcinoma-no special type (BCa) and corresponding metastatic lymph nodes ( $\mathrm{mLNs}$ ) and to compare the expression of these markers in $\mathrm{BCa}$ with and without metastasis using clinical samples.

\section{Methodology}

Tissues of 24 metastatic BCa (mBCa) with mLNs and 17 non metastatic BCa (nmBCa) obtained from the Department of Pathology, Faculty of Medicine, Peradeniya were included. Immunohistochemical expression of E-cadherin (epithelial cell adhesion membrane marker) and vimentin (mesenchymal cytoplasmic marker) was observed on all BCas and $\mathrm{mLNs}$. Ten random high-power fields $(10 \times 40)$ of 100 cells each were assessed to determine the percentage of positive cells. The mean expression of markers was compared between $\mathrm{mBCa}$ and $\mathrm{nmBCa}$ by independent sample T-test and $\mathrm{mBCa}$ and $\mathrm{mLN}$ by paired sample T-test.

\section{Results}

The mean E-cadherin expression was $70.54 \%$ (27.67\%-97.67\%) in $\mathrm{mBCa}, 86.40 \%$ (63.33\%$96.67 \%$ ) in $\mathrm{nmBCa}$ and $89.95 \%$ (46.33\%-100\%) in $\mathrm{mLNs}$. The mean vimentin expression was $6.54 \%(2.37 \%-13.87 \%)$ in $\mathrm{mBCa}, 5.39 \%(2.12 \%-$ $9.77 \%)$ in $\mathrm{nmBCa}$ and $1.28 \%(0.13 \%-8.25 \%)$ in $\mathrm{mLNs}$. The E-cadherin expression was significantly lower in $\mathrm{mBCa}$ compared to $\mathrm{nmBCa}$ $(p=0.002)$ and in $\mathrm{mBCa}$ compared to $\mathrm{mLNs}$ $(p=0.000)$. Vimentin expression was not significant between $\mathrm{mBCA}$ and $\mathrm{nmBCa}$ $(p=0.176)$. Higher vimentin expression in $\mathrm{mBCa}$ compared to $\mathrm{mLNs}$ was statistically significant $(p=0.000)$.

\section{Discussion and conclusion}

The significantly lower expression of E-cadherin in $\mathrm{mBCa}$ compared to $\mathrm{nmBCa}$ suggests that loss of epithelial cell properties promotes metastasis. Significantly lower E-cadherin and significantly higher vimentin expressions in $\mathrm{mBCa}$ compared to $\mathrm{mLNs}$ is supportive of EMT in $\mathrm{BCa}$. The reversed pattern is noted in nodal $\mathrm{BCa}$ metastasis suggesting the possibility of mesenchymal-epithelial transition of metastatic cancer cells in the lymph node.

Financial assistance from University of

Peradeniya (RG/AF/2013/32/M) is

acknowledged. 